\section{Neurotoxicidade persistente secundária ao uso de lítio: relato de caso}

\author{
Persistent neurotoxicity secondary to \\ lithium use: case report
}

Sr. Editor,

O lítio é classicamente usado no tratamento do transtorno bipolar do humor ( $\mathrm{TBH})$ e tem sido relacionado à neurotoxicidade, com seqüelas persistentes. Em 1987 , Adityanjee et al. relataram, pela primeira vez, o termo SILENT (Síndrome do Efeito Neurotóxico Irreversível por Lítio), que tem como critério diagnóstico a presença de seqüelas no Sistema Nervoso Central secundárias à litioterapia que persistem após dois meses cessada a administração do mesmo. ${ }^{1}$ Segue o caso de uma paciente com TBH que desenvolveu SILENT no curso do tratamento com lítio.

ZK, 22 anos, feminina, iniciou TBH em 1998, aos 15 anos, com um episódio de depressão maior. Nesta ocasião, foi medicada com venlafaxina de liberação lenta $(75 \mathrm{mg} /$ dia), apresentando virada maníaca após um mês de tratamento. Retirado o antidepressivo, melhorou espontaneamente e manteve-se eutímica, sem medicamentos, nos dois anos seguintes. Em 2000, aos 17 anos, teve outro episódio depressivo que, após três meses sem tratamento, evoluiu para episódio maníaco. Medicada com oxcarbazepina 600 mg/dia, houve recuperação por um ano. Em janeiro de 2002, suspendeu a medicação e, no final de 2003, aos 20 anos, desenvolveu um episódio maníaco com sintomas psicóticos. Nesta ocasião, recebeu diagnóstico de TBH e realizou exames laboratoriais, sendo introduzido lítio 900 mg/dia e olanzapina 10 mg/dia, com suspensão desta última um mês após estabilização do quadro. De janeiro de 2004 a meados de junho de 2005, encontrava-se eutímica em uso apenas de lítio $1.200 \mathrm{mg} / \mathrm{dia}$ (litemia de 0,7 mEq/L). No final de junho de 2005, desenvolveu gastroenterite associada à febre e, após cinco dias, apresentou ataxia cerebelar, disartria e dismetria. Avaliada em $1^{\circ}$ de julho, teve suspenso o lítio e colhida litemia, que se mantinha em 0,7 mEq/L. Em 21 de julho de 2005, submeteu-se à ressonância nuclear magnética (RNM) de crânio (normal) e iniciou fisioterapia, fonoterapia e natação (duas horas por semana). Em setembro de 2005, ainda mantinha síndrome cerebelar e, apesar de estar eutímica, foi introduzida oxcarbazepina $600 \mathrm{mg} /$ dia como medida profilática para o TBH. Evoluiu com melhora progressiva e, até janeiro de 2006, mantinha ataxia e disartria leves.

Existem cerca de 90 casos de SILENT publicados na literatu$\mathrm{ra}^{2}$ sendo a síndrome mais freqüente no sexo feminino (49 casos), com idades variando de 21 a 77 anos. Os fatores mais relacionados à síndrome são: febre, ${ }^{3}$ uso de antipsicóticos típicos e lesão cerebral. ${ }^{2}$ Embora ocorra mais freqüentemente com litemias elevadas, pode se desenvolver também com lítio na faixa terapêutica. A etiopatogenia é desconhecida, mas já foi detectada desmielinização em múltiplas regiões cerebrais, principalmente, no cerebelo. ${ }^{4}$

Este relato ilustra a ocorrência de um caso de SILENT, com envolvimento cerebelar em paciente do sexo feminino, como comumente relatado na literatura, porém com níveis terapêuticos de litemia, sem outros fatores de risco, exceto febre. Sendo assim, apesar da dosagem do lítio ser de extrema importância, vale ressaltar que a neurotoxicidade pode ser ocasionada ou facilitada por outros fatores, independentemente do valor da litemia, tornando-se um alerta para os profissionais que assistem essa população. ${ }^{2}$
Leandro Carvalho Araújo, Fabiana Nery-Fernandes, Lucas C Quarantini, Ângela Miranda-Scippa

Departamento de Neuropsiquiatria, Faculdade de Medicina, Universidade Federal da Bahia (UFBA), Salvador (BA), Brasil

Financiamento: Inexistente

Conflito de interesses: Inexistente

\section{Referências}

1. Schou M. Long-lasting neurological sequelae after lithium intoxication. Acta Psychiatr Scand. 1984;79(6):594-602.

2. Adityanjee, Munshi KR, Thampy A. The syndrome of irreversible lithium-effectuated neurotoxicity. Clin Neurofarmacol. 2005;28(1):38-49.

3. Sharma HS, Hoopes PJ. Hyperthermia induced pathophysiology of the central nervous system. Inj J Hyperthermia. 2003;19(3):325-54.

4. Donaldson IM, Cunninghan J. Persisting neurologic sequelae of lithium carbonate therapy. Arch Neurol. 1983;40(12):747-51.

\section{Limbic encephalitis manifesting as a psychotic disorder}

\author{
Encefalite límbica expressa como \\ transtorno psicótico
}

\section{Dear Editor,}

Several neurological diseases become apparent as a behavioral disorder that may be misunderstood as a primary psychiatric disorder. For instance, a series of degenerative (Huntington's disease), inflammatory (systemic lupus erythematosus), infectious (viral encephalitis, neurosyphilis), and metabolic disorders (porphyria, leukodystrophies) might have psychosis as their initial manifestation. ${ }^{1-3}$ Herein we report on a case of limbic encephalitis that has been misdiagnosed as a schizophreniform disorder with catatonic features.

A 40-year-old man with a history of psychotic symptoms and behavioral abnormalities for 6 months was referred to our department. According to his relatives, he was completely asymptomatic until the development of progressive behavioral changes. At onset he alternated periods of agitation and aggressiveness with periods of apathy. He also reported auditory and visual hallucinations as well as intense feelings of fear. He had been evaluated by a psychiatrist in the countryside and medicated with haloperidol $5 \mathrm{mg} /$ day. Despite treatment, he evolved with puerility and became very dependent for personal care. He had no previous history of substance abuse, psychiatric or neurological diseases.

His examination revealed a patient with obvious cognitive deficits (Mini-Mental Score Examination: 9/30 - the expected for 4 years of formal education is $25 / 30^{4}$ ), including memory and naming. No psychotic symptom was evident. He presented gegenhalten, generalized hyperreflexia and bilateral Babinski's sign. Abnormal involuntary movements such as grimacing and bruxism were also noticed. Based on his clinical history (one of progressive behavioral and cognitive decline) and physical findings, it was hypothesized that an organic mental disorder was presenting as a psychotic syndrome.

Laboratory work-up, including complete blood count, creatine kinase level, electrolytes, renal, liver and thyroid function tests, 